\title{
The Impact of Optical Factors on Resolution Acuity in Children with Down Syndrome
}

\author{
Julie-Anne Little, ${ }^{1}$ J. Margaret Woodbouse, ${ }^{2}$ Jan S. Lauritzen, ${ }^{1}$ and Katbryn J. Saunders ${ }^{1}$
}

Purpose. Down syndrome (DS) is associated with reduced visual acuity that cannot be explained by motivational or attentional factors. To isolate the contribution of optical factors to visual performance in DS, two types of resolution acuity were measured: grating resolution acuity, which is limited by optical quality, and interferometric acuity, which effectively bypasses the optics of the eye.

Methods. Twenty-nine children with DS (age range, 9-16 years) were tested. Sixty-eight age-matched, developmentally healthy children acted as controls. All wore best refractive correction, and none had clinically significant ocular disease. Grating resolution and interferometric acuity were measured according to a two-alternative, forced-choice procedure.

RESUlts. There was no change in grating resolution acuity or interferometric acuity with age in either group. Mean grating resolution acuities were $-0.12 \mathrm{log}$ of the minimum angle of resolution (logMAR; $\mathrm{SD} \pm 0.07$ ) for the control group and $+0.48 \log$ MAR (SD \pm 0.09 ) for the DS group. Mean interferometric acuities were $-0.11 \log$ MAR $(S D \pm 0.08)$ for the control group and +0.004 ( $S D \pm 0.06$ ) for the DS group. In the DS group, the mean interferometric acuity was reduced by a factor of 1.3, whereas mean grating resolution acuity was substantially reduced, by a factor of 4.1 , compared with controls.

Conclusions. Grating resolution and interferometric thresholds are reduced in children with DS. However the discrepancy between developmentally healthy children and those with DS is greater for grating resolution acuity, suggesting that degradation in optical quality is a major contributor to poor visual performance in children with DS. (Invest Ophthalmol Vis Sci. 2007;48:3995-4001) DOI:10.1167/iovs.06-1387

$\mathrm{D}$ own syndrome (DS) is associated with increased incidence of refractive error, ${ }^{1-4}$ strabismus, ${ }^{5}$ accommodation problems,${ }^{6-8}$ amblyopia, ${ }^{9}$ nystagmus, ${ }^{10}$ keratoconus, ${ }^{11}$ and other ocular disorders. Even with fully corrected refractive errors and in the absence of manifest abnormalities, children with DS have reduced visual acuity compared with their developmentally healthy peers. Woodhouse et al. ${ }^{12}$ used the Cardiff Acuity Test and Teller Acuity cards to measure visual acuity in 53 children with DS (age range, 12 weeks- 4.75 years) and compared results with those of age-matched control chil-

From the ${ }^{1}$ Vision Sciences Research Group, Biomedical Sciences, University of Ulster, Northern Ireland, United Kingdom; and the ${ }^{2}$ School of Optometry and Vision Sciences, Cardiff University, Cardiff, Wales, United Kingdom.

Supported by the Northern Ireland Department of Education and Learning (J.-A.L.).

Submitted for publication November 16, 2006; revised February 22 and April 19, 2007; accepted June 26, 2007.

Disclosure: J.-A. Little, None; J.M. Woodhouse, None; J.S. Lauritzen, None; K.J. Saunders, None

The publication costs of this article were defrayed in part by page charge payment. This article must therefore be marked "advertisement" in accordance with 18 U.S.C. $\$ 1734$ solely to indicate this fact.

Corresponding author: Julie-Anne Little, Vision Sciences Research Group, Biomedical Sciences, University of Ulster, Northern Ireland, United Kingdom; ja.little@ulster.ac.uk. dren. The study found that visual acuity in DS agrees well with age-matched controls from early infancy to 2 years of age and then falls below the normal range with increasing age. This finding was evident for children with and without clinically significant refractive error. Courage et al. ${ }^{13}$ also found reduced visual acuity in children with DS using Teller Acuity cards.

The etiology of poor visual performance recorded in DS is not fully understood. Previous authors have investigated whether motivation and attention deficits may reduce performance. For example, John et al. ${ }^{14}$ used behavioral and visualevoked potential (VEP) measures of visual acuity with the premise that VEP measures are relatively unaffected by motivation and attention factors. The investigators found that visual acuity thresholds were significantly poorer in the DS subject group in VEP and behavioral measures. Poor VEP performance indicated that visual deficits in DS could not be wholly attributed to motivation and attention and that a real sensory deficit exists. A neural deficit at least partly accounts for reduced visual acuity in DS, a suggestion supported by histologic reports that describe differences in the visual cortices of subjects with DS. These differences include lesser brain weights and less organized configuration of layers in the visual cortex in subjects with DS compared with controls. ${ }^{15}$ There is also evidence of dendritic atrophy and poor maturation. ${ }^{15,16}$ Further evidence for visual cortex deficits is presented by authors describing subtle discrepancies in pattern and flash VEP profiles in adults with DS. ${ }^{17,18}$ However, Ellingson ${ }^{19}$ demonstrated only mild and transient differences in flash VEPs in newborns with and without DS that disappear after 6 months of age.

Although VEP measures reflect the integrity of the visual pathway, up to and including the level of the primary visual cortex, behavioral measures of acuity are also influenced by higher centers of visual and cognitive processing. John et al. ${ }^{14}$ demonstrate a similar performance gap between VEP and behavioral acuity measures in a DS group and an age-matched control group. This suggests that the deficit in DS may occur at any point in the visual pathway up to the primary visual cortex but that it is not likely to be located at higher cortical areas. Optical, retinal, and neural factors may all be implicated, but attentional and motivational factors are not.

Few studies describe retinal structure in DS. Previous reviews of ocular findings report retinal anomalies or retinal abnormalities, mostly associated with high myopia, or incidences of retinal detachments. ${ }^{20-24}$ A few case studies cite instances of optic disc elevation, ${ }^{25,26}$ macular coloboma, ${ }^{27}$ Leber congenital amaurosis, ${ }^{28}$ and a possible link between DS and retinoblastoma. ${ }^{29,30}$ There is also evidence of increased vasculature at the optic nerve head. ${ }^{23,31-33}$ No studies have been published regarding retinal function in DS using techniques such as electroretinograms.

The optical components of the eye in DS are known to be at increased risk for abnormalities, including early-onset cataract, ${ }^{21}$ keratoconus, ${ }^{11,34}$ refractive error, ${ }^{1,3,35}$ and poor accommodative function. ${ }^{3,5}$ Poor optical quality has implications for retinal and cortical image quality.

The aim of the present study was to isolate the contribution of optical factors to visual performance in DS. It included interferometric acuity measurement, a technique used to as- 
sess retinal resolution, bypassing the optics of the eye by projecting a monochromatic sinusoidal grating pattern onto the retina, thus providing a resolution acuity measure minimally influenced by optical quality. Comparison of interferometric resolution acuity with psychophysical measurement of grating resolution acuity revealed the contribution of optical factors to visual performance in DS. No previous reports have been published describing interferometric grating acuity in DS, and few have been published in developmentally healthy children. 36,37

\section{Subjects ANd Methods}

\section{Subjects}

Recruitment and experimental protocols complied with the Declaration of Helsinki, and ethical approval was granted by local research ethics committees.

\section{Developmentally Healthy Subject (Control) Group}

The authors were granted permission from the Northern Ireland Education and Library Boards to approach schools for recruitment purposes. Local primary and secondary schools were contacted, and principals agreed to send out information leaflets and consent forms to all parents of children 9 to 16 years of age. Written informed consent was obtained from the parents of 71 (42\%) children. Three subjects were excluded because they did not meet inclusion criteria. Inclusion criteria were corrected visual acuity better than $0.15 \mathrm{log}$ of the minimum angle of resolution $(\operatorname{logMAR} ; 6 / 9$ approximate Snellen equivalent) and no history of amblyopia, squint, or ocular abnormality. For those subjects, a report was sent to parents or guardians recommending full eye examination. The 68 remaining subjects ranged in age from 9 to 16 years (mean age, $12.4 \pm 1.8$ years) and comprised 33 boys and 35 girls.

\section{Down Syndrome Subject Group}

Subjects with DS were members of the Cardiff University Down's Syndrome Vision Research Unit. Children with clinically detectable ocular conditions such as keratoconus, cataract, and retinal abnormality were excluded from participation. All remaining subjects aged 9 to 16 years in the cohort were contacted with information regarding the study. Written informed consent was obtained from parents of 29 (25\%) children. Participants ranged in age from 9 to 16 years (mean age, $12.84 \pm 1.9$ years) and comprised 19 boys and 10 girls.

\section{Procedure}

Testing for the control group took place in the subjects' schools, and testing for the DS group was carried out in the School of Optometry and Vision Sciences, Cardiff University. Test rooms were quiet and darkened. The same test apparatus was used across sites, under uniform testing conditions.

Refractive status was assessed using standard distance static retinoscopy, and spectacle correction was worn for all testing. Refractive errors were corrected if they exceeded $-0.25 \mathrm{DS},+1.25 \mathrm{DS}$, or astigmatism greater than $-0.50 \mathrm{DC}$. This was applied with each subject's own spectacles or with trial lenses. Trial lenses were used if spectacles were unavailable or did not provide the full or current prescription.

Monocular recognition acuity was measured at $3 \mathrm{~m}$ using the crowded $\log$ MAR acuity test. ${ }^{38}$ All subjects successfully completed acuity testing with either letter naming or letter matching.

Ocular dominance was ascertained for each subject using a typical finger-pointing method, based on the Bryngelson technique. ${ }^{39}$ Each subject's nondominant eye was patched, and pupil size of the dominant eye was assessed to ensure it was larger than $4 \mathrm{~mm}$ in diameter, the recommended minimum diameter for Lotmar interferometric acuity testing. Subsequently, two grating acuity tests-interferometric resolution acuity and grating resolution acuity-were applied in random order for all control and DS subjects.

\section{Interferometric Resolution Acuity}

Interferometric acuity was measured using the Haag-Streit Lotmar visometer mounted on a Haag-Streit slit lamp biomicroscope. Each subject, with chin on the slit lamp chin rest, viewed the target through a small aperture. The interferometer beam was directed through the center of the subject's pupil and was maintained in this position throughout testing. Light aperture was $0.5 \mathrm{~mm}$, and a green filter $(550$ $\mathrm{nm}$ ) was used. The visual field was set at $3.5^{\circ}$ centered on the fovea, and the light was set at $5 \mathrm{~V}$. This configuration is typical of Lotmar usage. ${ }^{40,41}$

The Lotmar visometer target is a circular sinusoidal grating that can be set at different spatial frequencies. When the spatial frequency is too high to resolve the target as a grating of a particular orientation, the target appears as a solid illuminated circle. The Lotmar visometer uses a decimal scoring system, from 0 to 2.5 on a continuous dial. A score of 1 indicates a spatial frequency of 30 cycles per degree; scores above this indicate higher spatial frequencies.

Horizontal and vertical diffraction gratings were presented to the subject in a randomized fashion. Pilot experiments using oblique, horizontal, and vertical grating orientations for both the grating resolution acuity technique and the interferometric acuity measures demonstrated that obliques proved more difficult for children to articulate and produced less repeatable thresholds; hence, only horizontal and vertical gratings were used. Before testing, an oral and diagrammatic explanation of the method was given. In this orientation discrimination task, the subject was asked to indicate whether the "stripes" or "lines" were running in a vertical or a horizontal direction. Some subjects used a matching card to indicate their choice of orientation. Many used hand gestures to signal "flat" or "standing" lines.

Target presentation in the Lotmar visometer is restricted to manual operation; therefore, a simple ascending staircase procedure was adopted to obtain a threshold. If a subject correctly identified at least three of four presentations, the interferometric grating was increased in spatial frequency by 0.1 decimal score and was repeated. Interferometric gratings grew progressively finer until subjects were unable to identify the orientation correctly on at least three of four presentations. At this point, the grating was made coarser by 0.05 decimal units; if the orientation was correctly identified (three of four times), acuity was recorded at this level. If not, acuity was recorded as the decimal score 0.05 below this level. Such ascending staircase procedures are commonly used for this type of data collection. ${ }^{37,42}$

\section{Grating Resolution Acuity}

Grating resolution acuity was measured using horizontal and vertical high-contrast sine wave gratings displayed side by side on a computer monitor (600 Multiscan; Sony, Tokyo, Japan). Each grating stimulus covered $512 \times 512$ pixels on the monitor. The subject's task was to indicate on which side of the screen the vertical sine wave grating was displayed (a two-alternative, forced-choice orientation discrimination task). The experimental protocol was implemented using the psychophysics toolbox in MatLab. ${ }^{43,44}$ A QUEST procedure was used to determine thresholds. ${ }^{45}$ QUEST is a highly efficient psychophysical method that calculates valid thresholds from a small number of trials. The fitting algorithm allows for $3 \%$ finger error, which means that any response of $97 \%$ or better is considered to fit the upper asymptote.

Subject responses were input by the examiner, allowing the next presentation to be displayed. Each trial contained 20 stimulus presentations. The method was explained in oral and diagrammatic form. All subjects were given at least one practice trial before testing began to ensure instructions were understood correctly. Testing distance was $3 \mathrm{~m}$ for controls and $1.5 \mathrm{~m}$ for DS subjects. If $1.5-\mathrm{m}$ thresholds approached the resolution limit of the monitor, testing distance could be increased to $3 \mathrm{~m}$; however, this situation did not arise in the DS group. 
For both procedures, the operator was not masked to the responses of the subject. This was necessary because the Lotmar visometer required manual operation. To ensure continuity, the operator was not masked to the grating resolution task either. However, the operator was positioned outside of the subject's field of view. In this way, subconscious cues from the operator were prevented. Encouragement was given after all responses, whether correct or incorrect. No further feedback was offered.

\section{Results}

\section{Success Rates}

Of the 68 control subjects, acuity measures were obtained from 66 (97\%) children for logMAR recognition acuity, 67 (99\%) children for interferometric acuity, and from 65 (95.6\%) children for grating resolution acuity.

Of the 29 children with DS, acuity measures were successfully obtained from 28 (96.6\%) children for logMAR recognition acuity, 26 (90\%) children for interferometric acuity, and 27 (93\%) children for grating resolution acuity. In both groups, those who were unable to complete testing were generally uncooperative.

\section{Refractive Error}

Haugen et al. ${ }^{5}$ studied refractive error longitudinally in children with DS and found few children showed the expected reduction in refractive error over time typical of developmentally healthy populations. They conclude there is a failure to emmetropize in $\mathrm{DS}^{5}$ (see also Cregg et al. $^{46}$ ). In the present study, refractive error data were recorded for each subject, and the subjects were fully corrected for testing. To examine any possible association between refractive error and grating resolution acuity in the DS group, Figure 1 plots the mean spherical equivalent refractive error against grating resolution acuity. The graph demonstrates no correlation between these two conditions $(r=-0.09 ; P=0.63)$.

\section{LogMAR Recognition Acuity}

Figure 2 plots $\log$ MAR recognition acuity for the control and the DS groups and indicates $0.00 \log$ MAR recognition acuity (6/6 Snellen equivalent; horizontal dashed line). LogMAR recognition acuity was significantly different between the two groups (two-sample independent $t$-test; $\mathrm{t}_{(92)}=-15.3 ; P<$

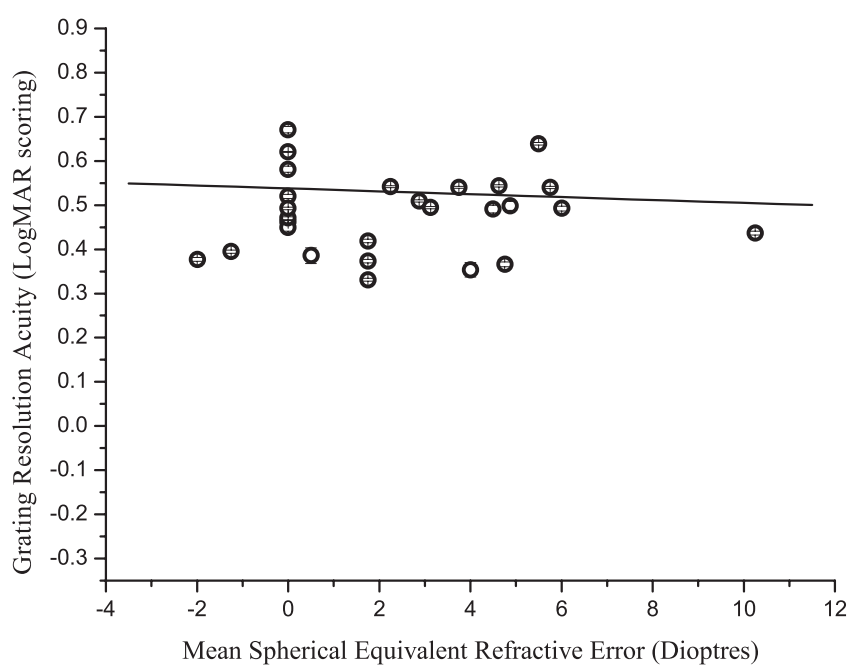

FIGURE 1. Scatterplot depicting mean spherical equivalent refractive error against grating resolution acuity for all subjects with Down syndrome. Linear regression is shown as a solid line.

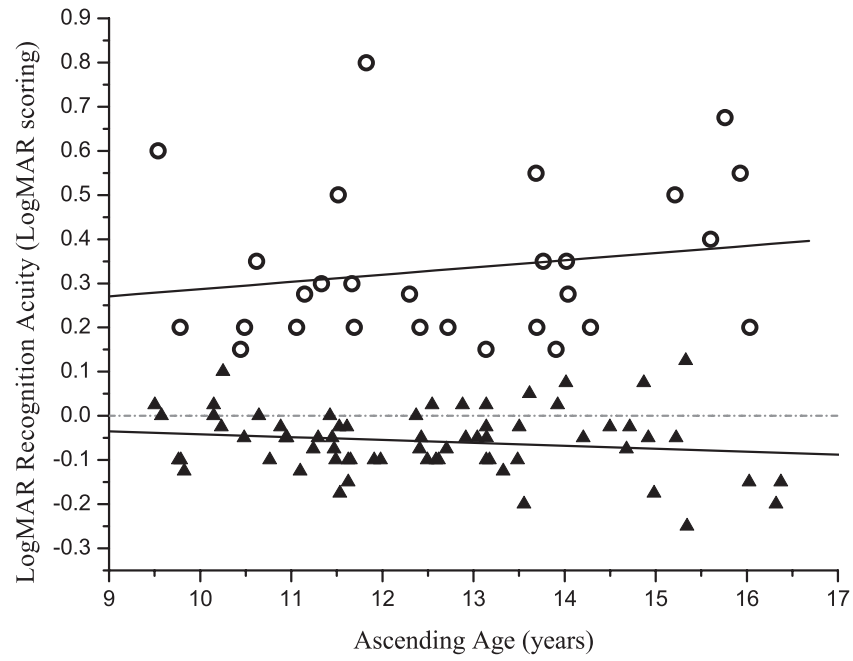

Figure 2. Recognition acuity expressed in $\log$ MAR units, plotted against each subject's age. Results are shown for children with DS $(\bigcirc)$ and developmentally healthy children $(\boldsymbol{\Lambda})$. Mean recognition (logMAR) acuity for the DS group is $+0.332 \operatorname{logMAR}, \mathrm{SD} \pm 0.18$. Mean logMAR acuity for the control group is $-0.06 \log$ MAR, $S D \pm 0.07$. Dashed line indicates 60 arc sec, equivalent to Snellen acuity of $6 / 6$. Solid lines indicate linear regression of the data.

0.0001). Linear regression revealed $\log$ MAR acuity to be invariant with age for both groups. Correlation coefficient values for these analyses are as follows: DS group, $r=0.18(P=0.36)$; control group, $r=-0.16(P=0.20)$.

\section{Grating Resolution Acuity}

Figure 3 plots grating resolution acuity against age for control and DS groups. Note that for grating resolution and interferometric acuity, performance was expressed in logMAR to compare with recognition acuity. Grating resolution acuity was significantly different between the two groups (two-sample independent $t$-test; $\left.\mathrm{t}_{(90)}=-35.6 ; P<0.0001\right)$. The difference in performance was strikingly large; children with DS had substantially poorer grating resolution acuity than their age-

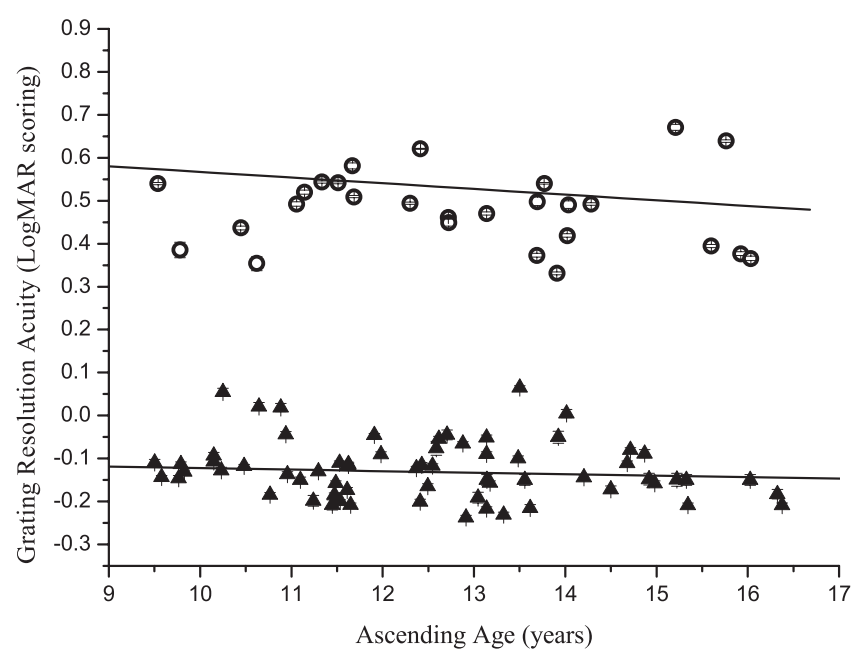

FIGURE 3. Grating resolution acuity expressed in logMAR units, plotted against subject's age. Results are shown for children with DS $(\bigcirc)$ and developmentally healthy children $(\boldsymbol{\Delta})$. Mean grating resolution acuity for the DS group was $+0.48 \log \mathrm{MAR}, \mathrm{SD} \pm 0.09$. Mean grating resolution acuity for the control group was $-0.12 \log$ MAR, SD \pm 0.07 . Solid lines indicate linear regression of the data. 
matched peers. For both groups, linear regression revealed no significant association between subject age and grating resolution acuity. Correlation coefficient values for these analyses are as follows: DS group, $r=-0.24(P=0.23)$; control group, $r=$ $-0.09(P=0.48)$.

\section{Interferometric (Retinal) Acuity}

Figure 4 plots the interferometric (retinal) acuity for the control and DS groups. For this measure of retinal acuity, there was a smaller deficit in the DS performance than in the control group. However, the difference was statistically significant (two-sample independent $t$-test; $\mathrm{t}_{(91)}=-7.6 ; P<0.0001$ ).

For both the groups, linear regression showed no significant association between subject age and interferometric resolution acuity. Correlation coefficient values for these analyses are as follows: DS group, $r=0.01,(P=0.97)$; control group, $r=$ $0.12(P=0.35)$.

The closeness of the control and DS group data is highlighted by the fact that acuity in six DS subjects lies within 1 SD from the control mean, and acuity in 16 DS subjects lies within 2 SD from the control mean. Ten DS subjects had interferometric acuities worse than 2 SD from the control mean values.

To compare the relative difference in performance between interferometric and grating resolution acuities in DS, a ratio was calculated by which individual acuities of DS subjects were divided by the relevant control mean, yielding a ratio revealing the relative performance of each child with DS compared with controls.

Figure 5 contains box-whisker plots of the mean of both acuity ratios for the DS group and, as reference, for the control group. Interferometric acuity in the DS group yielded visual performance that reflected most closely that of the control group. The ratio of the mean interferometric acuity was 1.3:1, whereas the grating resolution acuity mean ratio was $4.1: 1$, indicating grating resolution acuity was, on average, four times worse than normal in DS.

These findings were further supported by two-way analysis of variance (ANOVA) confirming a significant difference (at the $5 \%$ level) between the control and DS groups for all three measures $\left(\mathrm{F}_{(1,275)}=1022.7\right)$. Post hoc analysis (Scheffé test)

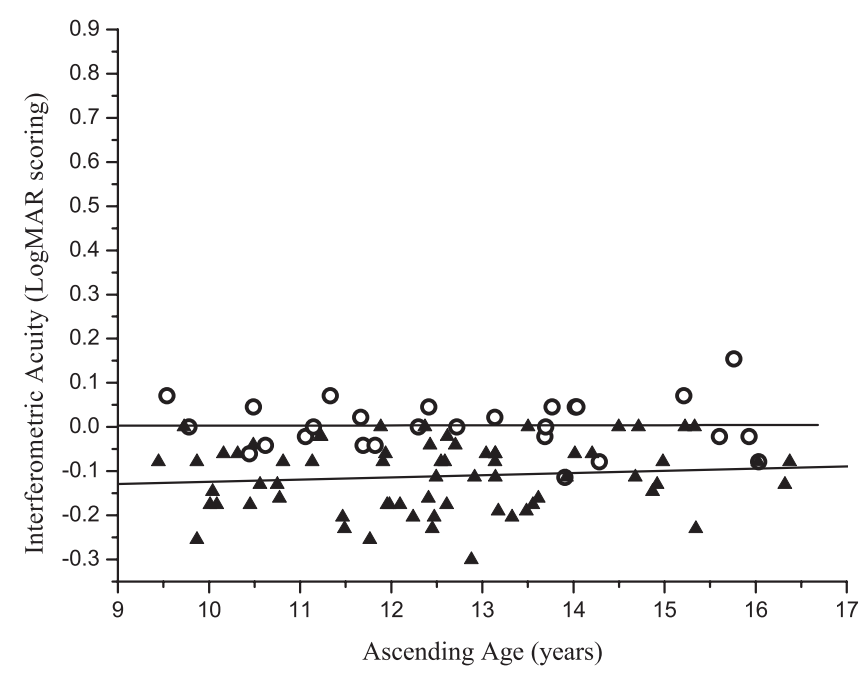

FiguRE 4. Interferometric retinal acuity, measured by the Haag Streit Lotmar visometer and expressed in logMAR units, plotted against subject age. Results are shown for children with DS (O) and developmentally healthy children $(\boldsymbol{\Delta})$. Mean interferometric acuity for the DS group was $+0.003 \log \mathrm{MAR}, \mathrm{SD} \pm 0.06$. Mean interferometric acuity for the control group was $-0.11 \log \mathrm{MAR}, \mathrm{SD} \pm 0.08$. Solid lines indicate linear regression of the data.

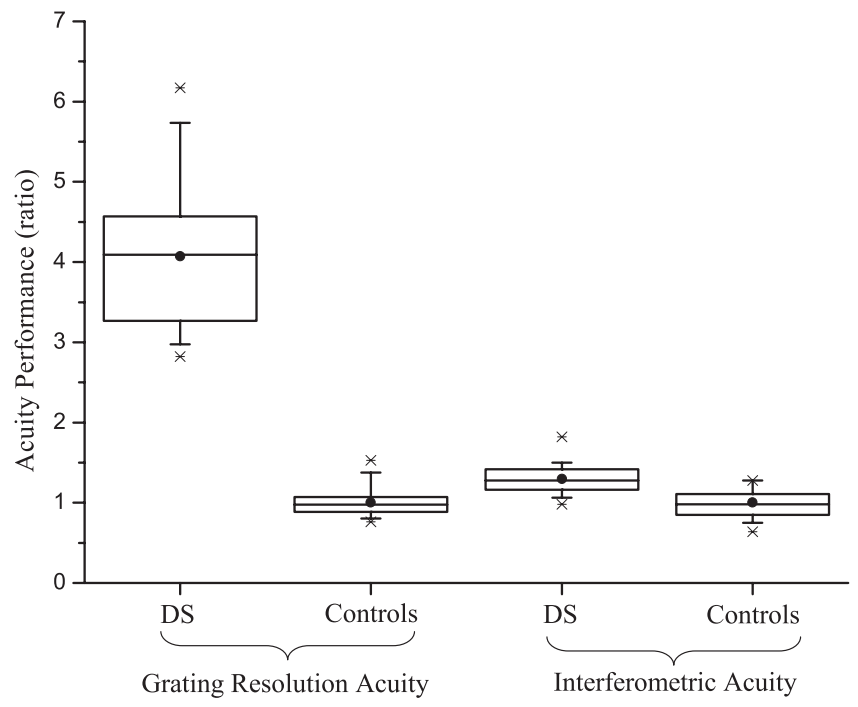

FIGURE 5. Box whisker plots of the mean DS and control group acuities. Acuities are expressed as a ratio of the measured acuity divided by the control mean acuity.

showed that the interactions between DS and the measures of recognition and resolution acuity were not significantly different (i.e., there was no significant difference in the amount by which these measures of acuity were degraded in the DS group). However, interferometric thresholds were significantly less elevated in the DS group (at the 5\% level) than were the other two measures of acuity.

\section{Modeling of Subject Responses}

To ensure that there was no systematic bias in the thresholds obtained by QUEST, experimental results for a simple ideal observer, with fixed chances to produce a false-negative value on any given suprathreshold trial, were modeled for a range of initial threshold guesses and underlying internal thresholds (-0.08, 0.22, 0.52, 0.7 logMAR).

For suprathreshold stimuli, the model observer had a $1 \%$ chance of producing a false-negative value during the first four trials (to account for the possibility of operator intervention). All subsequent suprathreshold presentations had a fixed $7 \%$ chance of producing a false-negative value. Based on the authors' experience, this represented a conservative estimate of response errors in both groups of children. For each internal threshold, 1000 runs were simulated. In each case, the measure of threshold slightly overestimated internal thresholds by an average of 0.01 logMAR, with a mean SD of $\pm 0.04 \operatorname{logMAR}$. Therefore, the model produced a measurement that slightly overestimated the underlying threshold.

\section{Discussion}

This is the first study concerning the influence of optical factors on resolution acuity performance in children with DS compared with their age-matched developmentally healthy peers. Interferometric acuity, which bypasses the eye's optics and reflects retinal integrity, was good in children with DS and in age-matched controls. A group mean of 61 arc sec was measured for the DS group, equivalent to Snellen acuity of $6 / 6$. However, in the grating resolution acuity task, visual performance of the DS group was severely degraded, indicating a preretinal source for the visual performance deficit in DS.

Retinal acuity, as assessed by interferometry, was close to normal levels in the DS group. However, a $t$-test revealed that 
a statistically significant difference between interferometric acuity measures in DS and control groups remained. No data on retinal function in DS and few reports of retinal structural anomalies exist. It is likely that retinal structure is largely intact and that the 1.3:1 reduction in interferometric acuity in DS indicates a deficit in cortical processing.

Grating resolution acuity is sensitive to optical blur and factors affecting stimulus resolution. In the present study, performance for the grating resolution acuity task was substantially worse for the DS group than for the control group and was relatively more degraded than interferometric acuity for each child with DS. These findings are supported by the post hoc analysis of the two-way ANOVA described in Results. The average grating resolution acuity was 4.1 times worse for a child with DS than for the age-matched equivalent.

Grating resolution acuity was better than logMAR recognition acuity in the control group, consistent with the hierarchical view in which recognition acuity is dependent on good resolution. ${ }^{47}$ However, in the DS group, though recognition and resolution acuity were significantly degraded (by factors of 2.6 and 4.1 , respectively), recognition acuity (mean +0.33 logMAR) was better than resolution acuity (mean +0.48 logMAR). Studies have shown grating resolution acuity to be particularly sensitive to small optical deficits, ${ }^{48}$ so this may be responsible for the observed discrepancy. Conversely, when the deficit is predominantly neural or retinal (e.g., amblyopia, age-related maculopathy), resolution acuity is less degraded than recognition acuity. ${ }^{49-51}$

Poor resolution acuity in subjects with DS suggested that optical limitations played a major role in poor visual performance, whereas relatively good interferometric acuity measures indicated well-preserved functional integrity in the retinas of the DS subjects.

Even subtle optical deficits in children with DS may influence the ability to resolve and recognize targets, and, though the authors excluded subjects with clinically evident optical conditions, the integrity of the tears, cornea, and lens might have been compromised in all subjects. Vincent et al. ${ }^{52} \mathrm{dem}-$ onstrate corneal videokeratography patterns consistent with keratoconus in some subjects with DS who showed no clinical signs of keratoconus. Structural evidence exists that the crystalline lens is thinner in persons with DS. ${ }^{5}$ Decreased central corneal thickness 5,53 and increased keratometry values in children with DS, indicating steeper corneas, ${ }^{5,52}$ have been reported. Differences in tear film integrity have been documented, ${ }^{54}$ with reduced numbers of goblet cells in the bulbar conjunctiva. ${ }^{55}$

The primary concern in designing the methods used to obtain acuity measures was efficient, rapid testing and simplicity of instruction and response. This was vital to maximize cooperation and success in what could be considered a challenging subject group. For these reasons, QUEST was chosen for the computer-based technique to assess grating resolution acuity. This option was not available for the interferometric acuity measure. However, interferometry is conducted at close range, making maintenance of attention less problematic than the relatively longer test distances required for the grating resolution task. Different psychophysical procedures are known to yield different estimates of the true underlying threshold measured; however, every possible effort was made to minimize these effects. Both tasks were deliberately designed with similar targets (horizontal and vertical gratings), and both tasks required the subject to identify orientation. Differences found in the results are too large to be explained purely by methodological differences.

The authors consider that both techniques provide measures of resolution acuity. However, it has been suggested that 2AFC procedures may be considered to yield detection thresholds, ${ }^{47}$ such as when a subject sees one orientation better than another and is able only to detect the location of the grating at the more visible orientation. However, at high spatial frequency, the mean luminance of horizontal and vertical gratings were measured and found to be equal $\left(21.4 \mathrm{~cd} / \mathrm{m}^{2}\right)$ to a precision of $0.1 \mathrm{~cd} / \mathrm{m}^{2}$. The actual contrast displayed on screen was 97\% (measurements taken with a colorimeter [PR-650 SpectraScan; Photo Research, Chatsworth, CA]). Subjects were instructed to attend both screen locations before choosing which contained the vertical rather than the horizontal grating. Under these circumstances, horizontal gratings would be more difficult to discern if meridional amblyopia existed. Refractive error and keratometry data do not support the presence of meridional amblyopia in the DS (or control) group.

For QUEST, beta (the notional threshold level of the psychometric function) was set at $\mathbf{8 1 \%}$. This is a typical value used for QUEST and is slightly higher than the convergence level of the interferometric task (approximately 75\%). QUEST procedures are known to systematically overestimate thresholds. A simple ideal observer model (see Modeling section in Results) shows that, in the present study, this overestimates thresholds by a negligible $0.01 \log$ MAR, which will, in any case, affect the DS and control group results equally.

It is known that early false negatives disproportionately elevate the final threshold. The operator was not masked to the correct response of the subject, so she could monitor whether an early false-negative response was influencing the quality of the test. This was addressed by restarting the test if an obvious early false negative occurred. In addition, the operator inspected the threshold plot after the test to ensure there were reversals within the test and that the plot appeared to converge to threshold. Measuring thresholds when the operator is unmasked to the correct response may introduce unintentional bias. In the present study, it is unlikely that any such bias influenced the differences between grating resolution and interferometric acuity because both measures across both subject groups were obtained by an unmasked operator. Furthermore, the operator was located outside the visual field of the subject, ensuring no subconscious cues were given.

Children with DS are known to have an increased prevalence of accommodative dysfunction. ${ }^{5-7} \mathrm{Did}$ accommodative inaccuracies limit the performance of children with DS in this study? Clinical records demonstrate that of the 29 subjects with DS, $41 \%$ had accurate accommodative responses. Working distances were $1.5 \mathrm{~m}$ for grating resolution acuity and $3 \mathrm{~m}$ for recognition acuity. A $1.5-\mathrm{m}$ test distance required less than one diopter of accommodation through a distance prescription, whereas the recognition task required half this amount. Such small accommodative demands render reduced accommodation an unlikely limitation to performance. Interferometric (retinal) acuity uses a technique to project the image directly onto the retina, bypassing the optics of the eye, which effectively makes the task independent of accommodative effort. It is therefore unlikely that accommodative inaccuracies limit performance in this task.

Given that refractive errors are persistent in DS when visual acuity is developing, an association between refractive error and grating resolution acuity in the DS group was considered. However, though it might be anticipated that poor grating resolution acuity results could be associated with higher refractive errors, this was not the case. There was no relationship between refractive error and grating resolution acuity performance (Fig. 1). It may be argued that this cohort of children with DS, by nature of their participation in an ongoing visual study, had obtained better than average optometric care and were less likely to have refractive amblyopia, which may be a factor in the general DS population. In the present study, poor performance in the grating resolution task cannot be attributed to refractive amblyopia. 
The present study has a number of strengths. A single examiner conducted all testing in a standardized manner after pilot studies in adults. The careful design of testing protocols, sensitive to the difficulties and challenges inherent in the subject group, ensured a high success rate such that more than $95 \%$ of DS and control children completed all tests. Thus the data collected across a range of ages and abilities are likely to accurately reflect performance in children with and without DS. Relatively few groups are working in the field of visual function in DS, and the present study adds to and supports existing data on visual performance in DS. The study provides the first investigation of interferometric acuity in DS. Finally, the control group data yield novel normative information on interferometric acuity in developmentally healthy children over a large age range.

The results of the present study support and enhance previous findings of visual acuity in DS, demonstrating a general deficit in visual acuity. ${ }^{12,13}$ This deficit is most marked for grating resolution acuity rather than interferometric acuity, which bypasses the optics of the eye. Grating resolution acuity was on average four times poorer than in age-matched controls, suggesting that optical quality has major implications for poor visual performance in DS. Further investigation into blur and optical integrity in children with DS is warranted.

\section{References}

1. Caputo AR, Wagner RS, Reynolds DR, Guo SQ, Goel AK. Down syndrome: clinical review of ocular features. Clin Pediatr (Phila). 1989;28:355-358.

2. Woodhouse JM, Pakeman VH, Cregg M, et al. Refractive errors in young children with Down syndrome. Optom Vis Sci. 1997;74: 844-851.

3. Woodhouse JM, Cregg M, Gunter HL, et al. The effect of age, size of target, and cognitive factors on accommodative responses of children with Down syndrome. Invest Ophthalmol Vis Sci. 2000; 41:2479-2485.

4. van Splunder J, Stilma JS, Bernsen RM, Arentz TG, Evenhuis HM. Refractive errors and visual impairment in 900 adults with intellectual disabilities in the Netherlands. Acta Opbthalmol Scand. 2003;81:123-129.

5. Haugen $\mathrm{OH}$, Hovding G. Strabismus and binocular function in children with Down syndrome: a population-based, longitudinal study. Acta Opbthalmol Scand. 2001;79:133-139.

6. Woodhouse JM, Meades JS, Leat SJ, Saunders KJ. Reduced accommodation in children with Down syndrome. Invest Ophthalmol Vis Sci. 1993;34:2382-2387.

7. Cregg M, Woodhouse JM, Pakeman VH, et al. Accommodation and refractive error in children with Down syndrome: cross-sectional and longitudinal studies. Invest Ophthalmol Vis Sci. 2001;42:5563.

8. Stewart RE, Woodhouse JM, Trojanowska LD. In focus: the use of bifocal spectacles with children with Down's syndrome. Ophthalmic Pbysiol Opt. 2005;25:514-522.

9. Tsiaras WG, Pueschel S, Keller C, Curran R, Giesswein S. Amblyopia and visual acuity in children with Down's syndrome. $\mathrm{BrJ}$ Opbthalmol. 1999;83:1112-1114.

10. Wagner RS, Caputo AR, Reynolds RD. Nystagmus in Down's syndrome. Ophthalmology. 1990;97:1439-1444.

11. Doyle SJ, Bullock J, Gray C, Spencer A, Cunningham C. Emmetropisation, axial length, and corneal topography in teenagers with Down's syndrome. Br J Ophthalmol. 1998;82:793-796.

12. Woodhouse JM, Pakeman VH, Saunders KJ, et al. Visual acuity and accommodation in infants and young children with Down's syndrome. J Intellect Disabil Res. 1996;40(pt 1):49-55.

13. Courage ML, Adams RJ, Reyno S, Kwa PG. Visual acuity in infants and children with Down syndrome. Dev Med Child Neurol. 1994; 36:586-593.

14. John FM, Bromham NR, Woodhouse JM, Candy TR. Spatial vision deficits in infants and children with Down syndrome. Invest Ophthalmol Vis Sci. 2004;45:1566-1572.
15. Becker L, Mito T, Takashima S, Onodera K. Growth and development of the brain in Down syndrome. Prog Clin Biol Res. 1991; 373:133-152.

16. Takashima S, Iida K, Mito T, Arima M. Dendritic and histochemical development and ageing in patients with Down's syndrome. $J$ Intellect Disabil Res. 1994;38(pt 3):265-273.

17. Kakigi R, Oono S, Matsuda Y, Kuroda Y. Pattern-reversal visual evoked potentials in Down's syndrome. Acta Neurol Scand. 1993; $87: 410-415$.

18. Fenton GW, Jones A, Forbes A. P313 evoked potentials in Down's syndrome. Electroencephalogr Clin Neuropbysiol. 1996;99(4): 354.

19. Ellingson RJ. Development of visual evoked potentials and photic driving responses in normal full term, low risk premature, and trisomy-21 infants during the first year of life. Electroencephalogr Clin Neurophysiol. 1986;63:309-316.

20. Ahmad A, Pruett RC. The fundus in mongolism. Arch Opbthalmol. 1976;94:772-776.

21. Roizen NJ, Mets MB, Blondis TA. Ophthalmic disorders in children with Down syndrome. Dev Med Child Neurol. 1994;36:594-600.

22. da Cunha RP, Moreira JB. Ocular findings in Down's syndrome. Am J Ophthalmol. 1996;122:236-244.

23. Berk AT, Saatci AO, Ercal MD, Tunc M, Ergin M. Ocular findings in 55 patients with Down syndrome. Opbthalmic Genet. 1996;17: 15-19.

24. Liza-Sharmini AT, Azlan ZN, Zilfalil BA. Ocular findings in Malaysian children with Down syndrome. Singapore Med J. 2006;47: 14-19.

25. Catalano RA, Simon JW. Optic disk elevation in Down's syndrome. Am J Opbthalmol. 1990;110:28-32.

26. Al-Hemidan AI, Al-Hazzaa SA, Chavis $\mathrm{P}, \mathrm{Al}-H u s s e i n ~ H$. Optic disc elevation in Down syndrome. Opbthalmic Genet. 1999;20:45-51.

27. Yamaguchi K, Tamai M. Congenital macular coloboma in Down syndrome. Ann Opbthalmol. 1990;22:222-223.

28. Hayasaka S, Noda S, Setogawa T, Tagusa Y, Kishida K. Leber congenital amaurosis in an infant with Down syndrome. Ann Opbthalmol. 1992;24:250-252.

29. Satge D, Gembara P, Sasco AJ, et al. An infant with Down syndrome and retinoblastoma: a possible non-fortuitous association. Opbthalmic Genet. 2001;22:117-123.

30. Brichard B, Vermylen C, De Potter P, Casteels I. Down syndrome: possible predisposition to retinoblastoma. Med Pediatr Oncol. 2003;41:73-74.

31. Williams EJ, McCormick AQ, Tischler B. Retinal vessels in Down's syndrome. Arch Opbthalmol. 1973;89:269-271.

32. Sherk MC, Williams TD. Disc vascularity in Down's syndrome. Am J Optom Physiol Opt. 1979;56:509-511.

33. Jaeger EA. Ocular findings in Down's syndrome. Trans Am Ophthalmol Soc. 1980;78:808-845.

34. Catalano RA. Down syndrome. Surv Opbthalmol. 1990;34:385398.

35. Haugen $\mathrm{OH}$, Hovding $\mathrm{G}$, Lundstrom I. Refractive development in children with Down's syndrome: a population based, longitudinal study. BrJ Ophthalmol. 2001;85:714-719.

36. Richman JE, Kozol N, Crawford RD. Use of interferometry in preschool children. J Am Optom Assoc. 1989;60:357-360.

37. Vernon SA, Hardman-Lea S, Rubinstein MP, Snead MP. White light interferometry in amblyopic children-a pilot study. Eye. 1990; 4(pt 6):802-805.

38. McGraw PV, Winn B. Glasgow acuity cards: a new test for the measurement of letter acuity in children. Opbthalmic Pbysiol Opt. 1993;13:400-404

39. Fink WH. The dominant eye: its clinical significance. Arch $O p b$ thalmol. 1938;19:555-582.

40. Bradley A, Thibos L, Still D. Visual acuity measured with clinical Maxwellian-view systems: effects of beam entry location. Optom Vis Sci. 1990;67:811-817.

41. Spurny RC, Zaldivar R, Belcher CD 3rd, Simmons RJ. Instruments for predicting visual acuity: a clinical comparison. Arch Opbthalmol. 1986;104:196-200. 
42. Geddes LA, Patel BJ, Bradley A. Comparison of Snellen and interferometer visual acuity in an aging noncataractous population. Optom Vis Sci. 1990;67:361-365.

43. Brainard DH. The psychophysics toolbox. Spat Vis. 1997;10:433436.

44. Pelli DG, Zhang L. Accurate control of contrast on microcomputer displays. Vision Res. 1991;31:1337-1350.

45. Watson AB, Pelli DG. QUEST: a Bayesian adaptive psychometric method. Percept Psychophys. 1983;33:113-120.

46. Cregg M, Woodhouse JM, Stewart RE, et al. Development of refractive error and strabismus in children with Down syndrome. Invest Ophthalmol Vis Sci. 2003;44:1023-1030.

47. Thibos LN, Bradley A. New methods for discriminating neural and optical losses of vision. Optom Vis Sci. 1993;70:279-287.

48. Han SH, Kim SK, Lee JB, Lee MS. The effects of diffusion blur on snellen and grating acuity and foveal function in amblyopia. Yonsei Med J. 1998;39:363-366.

49. Mayer DL. Acuity of amblyopic children for small field gratings and recognition stimuli. Invest Opbthalmol Vis Sci. 1986;27:11481153.

50. Friendly DS, Jaafar MS, Morillo DL. A comparative study of grating and recognition visual acuity testing in children with anisome- tropic amblyopia without strabismus. Am J Ophthalmol. 1990; 110:293-299.

51. White JM, Loshin DS. Grating acuity overestimates Snellen acuity in patients with age-related maculopathy. Optom Vis Sci. 1989;66: $751-755$.

52. Vincent AL, Weiser BA, Cupryn M, Stein RM, Abdolell M, Levin AV. Computerized corneal topography in a paediatric population with Down syndrome. Clin Exp Ophthalmol. 2005;33:47-52.

53. Evereklioglu C, Yilmaz K, Bekir NA. Decreased central corneal thickness in children with Down syndrome.J Pediatr Opbthalmol Strabismus. 2002;39:274-277.

54. Filipello M, Scimone G, Cascone G, Zagami A, Pantaleoni G. Ferning test in Down's syndrome. Acta Opbthalmol (Copenb). 1992; 70:274-277.

55. Filippello M, Cascone G, Zagami A, Scimone G. Impression cytology in Down's syndrome. Br J Ophthalmol. 1997;81:683-685.

56. Chylack LT Jr, Jakubicz G, Rosner B, et al. Contrast sensitivity and visual acuity in patients with early cataracts. $J$ Cataract Refract Surg. 1993;19:399-404.

57. McGraw PV, Barrett BT. Assessing retinal/neural function in the presence of ocular media opacities. Graefes Arch Clin Exp Ophthalmol. 1996;234:280-283. 